Methods: 140 PsA patients were at baseline examined with dual energy $x$-ray absorptiometry (DXA) with measurements at femoral neck and spine (L1-4). Trained nurses performed the DXA scans. The DXA machines used at baseline (Lunar Prodigy) and at 5 year follow-up (Lunar iDXA) were stable over the measurement periods. Cross calibration using a spine phantom was performed between the two DXA machines (BMD of the old phantom in the new machine and the old phantom in the old machine, with 55 measurement over a time period of 2 weeks) had a difference of less than 1\%. Demographic, disease measures and treatment data was collected at the same day or within 14 days to the date of DXA assessment. For group comparison, we used paired student t-test.

Results: After 5 years, 114 PsA patients (50.4\% women) were re-examined. Baseline mean (SD) was for age 51.4 (9.4) years, BMI 28.1 (3.4) kg/m2, disease duration 9.1 (6.7) years.

Disease characteristics at baseline and follow-up presented as mean (SD) were: DAS28 3.05 (1.1) and 2.4 (0.8); CRP 4.3 (6.7) and 5.1 (12.2) mg/dl, ESR 14.9 (11.1) and $10.9(12.9) \mathrm{mm} / \mathrm{hr}$; mHAQ $0.40(0.35)$ and $0.38(0.37)$.

The proportions of patients as baseline and follow up using conventional synthetic disease modifying anti-rheumatic drugs (DMARD) were $61.4 \%$ vs. $46.5 \%$; for biological DMARDs $37.7 \%$ vs. $48.2 \%$; for prednisolone $5.3 \%$ vs. $10.5 \%$, for targeted osteoporosis medication $0.9 \%$ vs. $3.5 \%$; for calcium and vitamin-D $11.4 \%$ vs. $26.3 \%$. As shown in the table, no significant change in femoral neck and spine BMD and T-scores was found for the 114 patients. However for Z-scores (age and weight adjusted T-score) a significant increase was found both at femoral neck and lumbar spine. When gender was examined separately no significant reduction in bone density was found in men whereas in women a significant reduction during follow up was only found for right femoral neck BMD.

\begin{tabular}{llll}
\hline & Baseline & 5 years & P value \\
\hline BMD spine L1-4 & $1.22(0.17)$ & $1.23(0.17)$ & 0.13 \\
T-score spine L1-4 & $0.21(1.42)$ & $0.32(2.01)$ & 0.42 \\
Z-score spine L1-4 & $0.20(1.32)$ & $0.78(2.05)$ & $<0.001$ \\
BMD Femoral neck L & $0.98(0.14)$ & $0.97(0.14)$ & 0.09 \\
T-score Femoral neck L & $-0.59(1.15)$ & $-0.50(1.02)$ & 0.14 \\
Z-score Femoral neck L & $-0.06(1.02)$ & $0.37(1.04)$ & $<0.001$ \\
BMD Femoral neck R & $0.98(0.14)$ & $0.98(1.16)$ & 0.57 \\
T-score Femoral neck R & $-0.59(1.10)$ & $-0.52(0.94)$ & 0.22 \\
Z-score Femoral neck R & $-0.08(1.02)$ & $0.35(0.90)$ & $<0.001$ \\
\hline
\end{tabular}

Conclusion: No reduction in BMD at femoral neck and spine was found in our PsA outpatient clinic cohort at baseline or over 5 years follow up. Rather, we found a statistically significant increase in age adjusted bone density (Z-score) both at femoral neck and spine over time. Our data thus adds evidence that PsA patients treated in ordinary clinical practice do not seem to be at an increased risk of developing osteoporosis.

REFERENCES:

[1] Tai-li Chen, et al. Bone Mineral Density, Osteoporosis, and Fracture Risk in Adult Patients with Psoriasis and Psoriatic Arthritis: A systematic Review and Meta-Analysis of Observational Studies, J Clin Med 2020;9:3712

[2] Agnete Malm Gulati, et al. Osteoporosis in psoriatic arthritis: a cross-sectional study of an outpatient clinic population. RMD Open 2018;4:1

Acknowledgements: Research nurse Hanne Vestaby and osteoporosis nurses at -Division of Rheumatology, Kristiansand.

Disclosure of Interests: Serina Brådland: None declared, Arthur Kavanaugh: None declared, Glenn Haugeberg Grant/research support from: Unrestricted Research Grant from Pfizer Norway.

DOI: 10.1136/annrheumdis-2021-eular.4038

\section{POS1086 DIFFERENCES IN PROINFLAMMATORY AND LIPID PROFILE BETWEEN PSORIATIC ARTHRITIS, ANKYLOSING SPONDYLITIS AND RHEUMATOID ARTHRITIS}

K. Bonek ${ }^{1}$, E. Kuca-Warnawin ${ }^{2}$, M. Ciechomska ${ }^{2}$, P. Głuszko ${ }^{1}$, E. Kontny ${ }^{2}$ ${ }^{1}$ National Institute of Geriatrics, Rheumatology and Rehabilitation, Department of Rheumatology, Warszawa, Poland; ${ }^{2}$ National Institute of Geriatrics, Rheumatology and Rehabilitation, Department of Pathophysiology and Immunology, Warsaw, Poland

Background: Patients with rheumatic diseases such as rheumatoid arthritis (RA), psoriatic arthritis (PsA), and ankylosing spondylitis (AS) are at increased risk of developing dyslipidaemia and premature cardiovascular disease (CVD)(1). Objectives: To investigate the relationship between proinflammatory cytokines, microRNA, and lipid profile in patients with RA, AS, and PsA

Methods: A group of 65 patients (RA15/ AS25/ PsA25) with high disease activity (mean DAS28 5,98 / ASDAS-CRP 3,7/ DAPSA 38,5) and 25 healthy controls (HC) were compared. Lipid profile comprised triglycerides (TG), total cholesterol(TC), low (LDL), and high-density lipoprotein (HDL). Serum concentrations of IL-6, IL-21,
IL-17, TNF and osteoprotegerine (OPG) were measured by commercially available enzyme-linked immunosorbent assays. Expression of miR-233-5p,miR-92-3p,miR 485-3p, miR-10b-5p,let-7d-5p, miR-26 -a-2-3p levels in sera was normalized to miR-16a internal control. The Mann-Whitney test was applied for intergroup comparison, the correlation was assessed using Spearman's Rank test

Results: Patients with RA revealed mixed dyslipidemia (mean values:TC196; LDL 117; HDL, 48 TG; $124 \mathrm{mg} / \mathrm{dl}$ ), PsA revealed hypertriglyceridemia (TC 175; LDL 100; HDL 50; TG137 mg/dl) and in AS no specific profile was found (TC 179; LDL98; HDL55; TG103 mg/dl). Higher expression of miR-485was observed in patients with PsA (4,8-fold) and AS (5,9-fold) compared to RA and HC groups $(3,1$ and 1,5 -fold $p=0,02)$. Similarly, miR-26a revealed higher expression in patients with PSA (28,4-fold) and AS (21,5-fold) than in RA and HC (3,5 and $2,9$-fold $p<0,00)$. PsA patients had higher expression of miR-146b than patients with RA, AS and $\mathrm{HC}(40,9$-fold vs 12,6 vs 15,7 vs 3,4 -fold $p=0,002)$ and higher miR-10b ( 11,7 vs 1,4 vs 4,9 vs 1,7 -fold $p=0,004)$. Patients with RA showed higher expression of let7-d than patients with PsA, AS and $\mathrm{HC}$ (22-fold vs 1,8 vs 2,3 vs1,9 -fold $p=0,002)$. In PsA miR-92b expression correlated negatively with HDL levels $(r=-0,62 p=0,02)$ and positively with fasting glucose $(r=0,71 p<0,00)$. TG levels negatively correlated with TNF $(r=-0,47 p=0,01)$, IL-17 $(r=-0,49 p=0,01)$ and OPG $(r=-0,51 p=0,00)$ serum levels. Let-7d correlated negatively with TC $(r=-0,58 p=0,03)$. In RA IL-21 positively correlated with LDL $(r=0,71 p=0,00)$ and TC $(r=0,75 p=0,001)$ concentrations. TG levels correlated positively with expressions of miR-92b $(r=6,9 p=0,02)$ and miR-26a $(r=0,69 p=0,03)$. In AS expression of let-7d was correlated positively with $\operatorname{HDL}(r=0,41 p=0,00)$ and TC $(r=0,45$ $p=0,00)$ levels and negatively with ASDAS-CRP $(r=-0,675 p=0,02)$ and CRP levels $(r=-0,53 p=0,01)$. There were no significant differences in OPG, IL-21 concentrations or miR-146b, miR-92b,miR-233 expressions.

Conclusion: Differences in proinflammatory profile in RA, PSA, and AS seem to be associated with different phenotypes of dyslipidemia. In PsA expression of miR-92b and higher levels of TNF, IL-17 and OPG are associated with altered lipid profile and hypertriglyceridemia. High activity of AS is associated with lowered expression of let-7d, possibly influencing TC and HDL levels. Therefore, measuring lipid profile in active disease might be misleading. In active RA increase of TC and LDL levels was associated with high IL-21 concentration, while hypertriglyceridemia with miR-92b and miR-26a expressions.

\section{REFERENCES:}

[1] Bonek K,et al AB0775 Peripheral joint inflammation is associated with more proatherogenic cardiovascular risk profile in patients with psoriatic arthritis. Annals of the Rheumatic Diseases 2020;79:1685-1686

Disclosure of Interests: None declared.

DOI: 10.1136/annrheumdis-2021-eular.4072

\section{Osteoarthritis}

\begin{tabular}{|l|l}
\hline POS1087 & USING LIPIDOMICS TO PREDICT PREDNISOLONE \\
TREATMENT RESPONSE IN PATIENTS WITH \\
INFLAMMATORY HAND OSTEOARTHRITIS: THE HOPE \\
STUDY
\end{tabular}

M. Loef ${ }^{1}$, T. Faquih ${ }^{2}$, J. Von Hegedus ${ }^{1}$, M. Ghorasaini ${ }^{3}$, A. Ioan-Facsinay ${ }^{1}$, F. Kroon ${ }^{1}$, M. Giera ${ }^{3}$, M. Kloppenburg ${ }^{1,2}$. $^{1}$ Leiden University Medical Center, Rheumatology, Leiden, Netherlands; ${ }^{2}$ Leiden University Medical Center, Clinical Epidemiology, Leiden, Netherlands; ${ }^{3}$ Leiden University Medical Center, Center for Proteomics and Metabolomics, Leiden, Netherlands

Background: Lipidomics analysis has become a valuable technology for understanding patho-physiological mechanisms and may aid the identification of biomarkers of therapeutic responsiveness.

Objectives: To explore the use of lipidomics for prediction of prednisolone treatment response in patients with inflammatory hand osteoarthritis.

Methods: The Hand Osteoarthritis Prednisolone Efficacy (HOPE) study is a blinded, randomized placebo-controlled trial, that investigated the effect of prednisolone treatment in patients with painful, inflammatory hand $O A$, fulfilling the American College of Rheumatology criteria. The present analyses comprised only patients randomized to daily $10 \mathrm{mg}$ prednisolone treatment for six weeks. Response to prednisolone treatment was defined according to the OARSI-OMERACT responder criteria at six weeks. Baseline blood samples were obtained non-fasted. Lipid species were quantified in erythrocytes with the Lipidyzer ${ }^{\mathrm{TM}}$ platform (Sciex). After pre-processing of the data, 286 lipids species were available for further analyses $(\mathrm{nmol} / \mathrm{mL})$. In addition, we used an in-house LC-MS/MS platform to analyse oxylipins in plasma, identifying 25 oxylipins (area ratios). Elastic net regularized regression was used to predict prednisolone treatment response. A 10-fold cross-validation (CV) was performed for selection of the optimal tuning parameters based on the smallest CV mean prediction error. First, a model was fit with commonly assessed patient characteristics and patient reported outcomes, measured at baseline (model 1). Second, we fitted model 2 by adding the Lipidyzer ${ }^{\mathrm{TM}}$ platform lipids to model 1. Third, we fitted model 3 by adding the oxylipins to model 1. The discriminatory 
accuracy of the model was estimated by receiver operating characteristic (ROC) analyses. The area under the curve (AUC) and corresponding 95\% confidence intervals $(\mathrm{Cl})$ were calculated using 1,000 bootstrap replications. Results: Among the 40 patients included, 31 (78\%) fulfilled the OARSI-OMERACT responder criteria. From the included general patient characteristics (Table 1), elastic net selected baseline hand function as only predictor of treatment response, with an AUC of $0.78(95 \% \mathrm{Cl} 0.60 ; 0.96)$ (Figure 1). In model 2, we added the 286 Lipidyzer $^{\mathrm{TM}}$ platform variables to model 1. In addition to hand function, two lipids were selected: diacylglycerol(DAG)(16:0/16:0) and phosphatidylethanolamine(PE)(O-18:0/20:4), which improved the discriminatory accuracy to an AUC of $0.92(0.83 ; 1.02)$. Lastly, model 3 was fit with patient characteristics as well as oxylipins, resulting in selection of AUSCAN function and three oxylipin predictors: 9-hydroxy-octadecatrienoic acid (HOTrE), 5-hydroxy-eicosapentaenoic acid (HEPE) and 10-hydroxy-docosahexaenoic acid (HDHA), with an AUC of 0.85 (0.69;1.02). Conclusion: The patients' lipid profile improved the discriminative accuracy of the prediction of prednisolone treatment response in patients with inflammatory hand osteoarthritis compared to prediction by commonly measured patient characteristics alone. This exploratory study suggests that lipidomics is a promising field for biomarker discovery for prediction of anti-inflammatory treatment response.

Table 1. Baseline characteristics

\begin{tabular}{llll}
\hline & $\begin{array}{l}\text { All prednisolone } \\
\text { treated } \\
\mathrm{n}=40\end{array}$ & $\begin{array}{l}\text { Responders } \\
\mathrm{n}=31(78 \%)\end{array}$ & $\begin{array}{l}\text { Non- } \\
\text { responders } \\
\mathrm{n}=9(23 \%)\end{array}$ \\
\hline General characteristics & & & \\
Age, year & $62.4(9.3)$ & $62.9(9.4)$ & $60.8(9.4)$ \\
Sex, \% women & 85 & 84 & 89 \\
BMI, kg/m ${ }^{2}$ & $27.4(4.4)$ & $27.8(4.2)$ & $26.2(5.0)$ \\
Education, \% high & 46 & 42 & 56 \\
Disease duration & $6.7(7.1)$ & $7.2(7.4)$ & $4.9(5.8)$ \\
Erosive OA, \% & 71 & 74 & 56 \\
Kellgren-Lawrence sum score, 0-120 & $35.1(16.4)$ & $34.1(16.5)$ & $37.5(14.7)$ \\
Ultrasound synovitis sum score, 0-90 & $16.2(6.6)$ & $15.5(6.4)$ & $18.7(7.2)$ \\
VAS global assessment, 0-100 & $52.3(20.6)$ & $54.2(16.8)$ & $45.6(30.8)$ \\
AUSCAN pain, 0-20 & $11.0(3.3)$ & $11.3(2.4)$ & $10(5.4)$ \\
AUSCAN function, 0-36 & $17.7(7.6)$ & $19.6(6.6)$ & $11(7.5)$ \\
\hline
\end{tabular}

Numbers represent mean (SD) unless otherwise specified. AUSCAN = Australian/Canadian Hand Osteoarthritis Index, $\mathrm{BMI}=$ body mass index, VAS = visual analogue scale



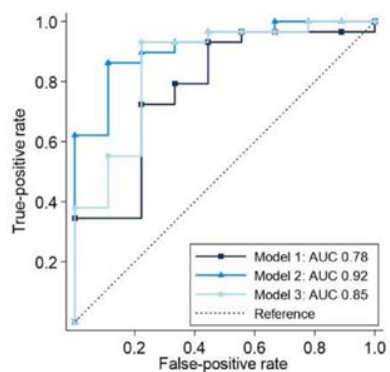

Figure 1. Prediction model characteristics

A) shows the variables included for model fitting of the three prediction models, colours correspond to the lines of the ROC curves in B). Of model 2, only the lipid classes are shown. Variables in bold font were selected in the final models. Model 1: General patient characteristics, model 2: model 1+ Lipidyzer ${ }^{T M}$ platform, model 3: model $1+$ oxylipin platform. Abbreviations: $\mathrm{AUC}=$ area under the curve, AUSCAN $=$ Australian $/$ Canadian $\mathrm{Han}$ Osteoarthritis Index, $C E=$ cholesteryl ester, $C E R=$ ceramide, $D A G=$ diacylglycerol, $D C E R=$ dihydroceramide, FFA = free fatty acid, HCER = hexosylceramide, $K L=$ Kellgren-Lawrence, $L$ CER = lactosylceramide, (L)PC (lyso) phosphatidylcholines, (L)PE = (lyso) phosphatidylethanolamine, $O A=$ osteoarthritis, $S M=$ sphingomyelin, TAG $=$ triacylglycerol, VAS $=$ visual analogue scale, 9-HOTrE $=9$-hydroxy-octadecatrienoic acid, 5-HEPE $=5$ hydroxy-eicosapentaenoic acid, 10-HDHA $=10$-hydroxy-docosahexaenoic acid.

Disclosure of Interests: Marieke Loef: None declared, Tariq Faquih: None declared, Johannes von Hegedus: None declared, Mohan Ghorasaini: None declared, Andreea loan-Facsinay: None declared, Féline Kroon: None declared, Martin Giera Shareholder of: Pfizer, Consultant of: Boehringer Ingelheim Pharma, Margreet Kloppenburg: None declared.

DOI: 10.1136/annrheumdis-2021-eular.19

\begin{tabular}{|l|l}
\hline POS1088 & EFFICACY OF SUBCUTANEOUS TANEZUMAB \\
& FOR THE TREATMENT OF OSTEOARTHRITIS OF \\
& THE KNEE OR HIP: A POST-HOC SUBGROUP \\
& ANALYSIS OF PATIENTS FROM A RANDOMIZED, \\
& NSAID-CONTROLLED STUDY WITH A HISTORY OF \\
& DEPRESSION, ANXIETY, OR INSOMNIA
\end{tabular}

P. J. Mease ${ }^{1}$, T. Mallick-Searle ${ }^{2}$, E. Johnston ${ }^{3}$, L. Viktrup ${ }^{3}$, D. Menuet ${ }^{4}$, R. Yang ${ }^{5}$, R. J. Fountaine ${ }^{6}{ }^{1}$ Swedish Medical Center \& Providence St. Joseph Health and University of Washington, -, Seattle, United States of America; ${ }^{2}$ Stanford Health Care, Redwood City, United States of America; ${ }^{3}$ Eli Lilly and Company, -,
Indianapolis, United States of America; ${ }^{4}$ Pfizer SAS, Paris, France; ${ }^{5}$ Pfizer Inc., New York, United States of America; ${ }^{6}$ Pfizer Inc., Groton, United States of America

Background: Tanezumab is a monoclonal antibody directed against nerve growth factor. It is in development for the treatment of moderate to severe chronic pain associated with osteoarthritis $(\mathrm{OA})$ in adult patients for whom other treatments are ineffective or not appropriate. Phase 3 clinical trials have demonstrated the efficacy of subcutaneous tanezumab versus placebo for pain and function outcomes over various timepoints. Largely similar change from baseline was demonstrated in an oral nonsteroidal anti-inflammatory drug (NSAID)-controlled study. ${ }^{1,2,3,4}$ The efficacy of some other OA therapies can be dampened in patients with depression, anxiety, or insomnia. $5,6,7$

Objectives: A post-hoc analysis to explore the efficacy of subcutaneous tanezumab after 16 weeks treatment, as compared to oral NSAID, in patients with OA and a history of depression, anxiety, or insomnia at baseline.

Methods: Subgroup analysis of data from a randomized, double-blind, dou ble-dummy, active-controlled phase 3 study of subcutaneous tanezumab $(2.5 \mathrm{mg}$ or $5 \mathrm{mg}$ every 8 weeks) vs twice daily oral NSAID in patients ( $\geq 18$ years) with radiographically-confirmed moderate to severe hip or knee OA (Kellgren-Lawrence grade $\geq 2$; NCT02528188). ${ }^{4}$ Co-primary efficacy endpoints were change from baseline to week 16 in Western Ontario and McMaster Universities OA Index (WOMAC*) Pain and Physical Function subscale scores (both $\geq 5 / 10$ at randomisation; increasing score indicates increasing pain/disability), and Patient's Global Assessment of OA (PGA-OA, $\geq 3 / 5$ at randomisation; increasing score indicates poorer condition). Enrolled patients had a history of inadequate pain relief with acetaminophen; inadequate pain relief with/intolerance to/contraindication to tramadol or opioids; or an unwillingness to take opioids. Patients were on a stable dose of NSAID for $\geq 30$ days before screening. Data are presented as least squares (LS) mean change from baseline to week 16 for the whole population and for subgroups of patients with/without a history of depression, anxiety, or insomnia at baseline. Exploratory statistical analysis was conducted by analysis of covariance. $P$ values were not adjusted for multiplicity. This exploratory posthoc analysis was not part of the pre-specified hypothesis testing plan or included in any sample size calculations; therefore, comparisons between treatment arms or patient subgroups should be interpreted with caution.

Results: Overall, 2996 patients were randomized and received at least one dose of study treatment (subcutaneous tanezumab $2.5 \mathrm{mg}$ : $\mathrm{n}=1002$; subcutaneous tanezumab $5 \mathrm{mg}$ : $\mathrm{n}=998$; oral NSAID: $\mathrm{n}=996$ ). In this population (comprising patients with or without a history of anxiety, depression or insomnia), all treatments were associated with notable and largely similar magnitude improvements in WOMAC Pain, WOMAC Physical Function, and PGA-OA at week 16 (Figure 1). Across treatment groups, differences in LS mean change from baseline in patients with

Figure 1: Co-primary efficacy endpoints after 16 weeks of treatment
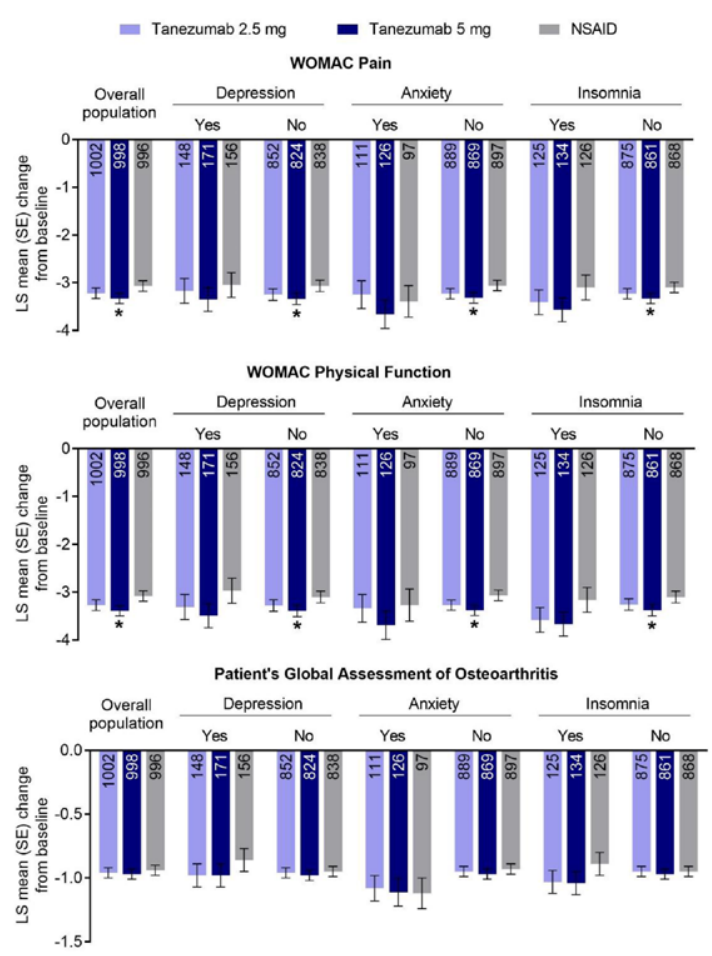

p $<0.05$ vS NSAID , NSAID, nonsteroidal anti-inflammatory drug; SE, standard error; WOMAC, Western Ontario and McMaster Universities Osteoarthritis Index.

*01996 Nicholas Bellamy. WOMAC ${ }^{\circ}$ is a registered trademark of Nicholas Bellamy (CDN, EU, USA) 